\title{
Host galaxies of 2MASS-selected QSOs to redshift $0.3^{1}$
}

\author{
J. B. Hutchings, N. Maddox \\ Herzberg Institute of Astrophysics, NRC of Canada, \\ Victoria, B.C. V9E 2E7, Canada; john.hutchings@nrc.ca \\ R. M. Cutri, B. O. Nelson \\ Caltech, IPAC, MS100-22, Pasadena, CA 91125
}

Received __; accepted _

\footnotetext{
${ }^{1}$ Based on observations made with the Canada France Hawaii telescope, which is operated by NRC of Canada, CNRS of France, and the University of Hawaii.
} 


\begin{abstract}
We present and discuss optical imaging of 76 AGN which represent the 2MASS-selected sample for $\mathrm{z}<0.3$, from a full list of 243 . They are found to have dust-obscured nuclei, residing in host galaxies that show a high fraction $(>70 \%)$ of tidal interactions. The derived luminosities of the AGN and host galaxies are similar to traditionally-selected AGN, and they may comprise some $40 \%$ of the total AGN population at low redshift. We have measured a number of host galaxy properties, and discuss their distributions and interrelations. We compare the 2MASS AGN with optically selected samples and the IRAS-selected galaxy samples, and discuss the differences in terms of merger processes and initial conditions.
\end{abstract}

Subject headings: galaxies: quasars: general; galaxies: interactions; galaxies: fundamental parameters; infrared radiation 


\section{Introduction}

The 2MASS survey has produced a sample of AGN which are not the traditional blue-selected objects (see e.g. Warren, Hewett, and Foltz 2000). The candidates were selected for colours redder than most known AGN (J-K>2.0), and the sample does not include any previously known AGN. Spectroscopic follow-up of the candidates has selected those with AGN spectra, which have been measured for redshifts. The optical spectra were obtained with a variety of telescopes, including the Palomar 5m, Keck, Steward 2.3m, and MSSO 2.3m (Cutri et al 2000). The AGN were classified, using standard line width and line ratio criteria, into types 1 and 2 and LINERS. Type 1 requires line widths of $10000 \mathrm{~km}$

$\mathrm{s}^{-1}$; some have both narrow and broad line components, leading to classifications between 1 and 2 .

A major question that arises is how these IR-selected AGN are related to the traditional blue-selected AGN. They may be dust-obscured versions of objects we are familiar with, or they may be a different population altogether. This paper describes a program to image low-redshift 2MASS AGN to compare their properties (in particular those of the host galaxies) with 'normal' AGN.

The sample chosen was the complete dataset of 2MASS-selected AGN with known redshift 0.3 or less, observable from the CFHT (declination between $-30^{\circ}$ and $60^{\circ}$ ). This list has 243 objects, with nuclear types well spread among types 1, 2, and LINERs. Radio fluxes at $1400 \mathrm{MHz}$ from the FIRST and NVSS catalogues, within 1.5 and 3.0 arcsec, respectively, were noted as associated. 


\section{Observations}

The observations were acquired as a CFHT 'snapshot' proposal, during the first semester of 2002. The list of 243 QSOs as described in the introduction, was used as the target list for 200-second integrations with the R-band filter and the CFHT 12K camera. The pixel sampling is 0.206". In almost all cases, a second 200-second exposure was obtained at the same time, to enable cosmic-ray rejection. A total of 205 such exposures were taken, along with a smaller number of 5 or 20 second exposures (a default to enable photometric standards to be measured elsewhere in the large CFHT 12K camera field). The QSOs were placed in the same CCD and position for all observations, so the detector properties are very uniform. The observing logs contained notes on the photometric quality and other variables at the time of observation. As the observations were taken over many different nights, there is some variation of observing conditions, and we were careful to investigate the effects of these on the derived results. Figure 1 shows the principal observational variations in the database.

Most of the images have FWHM in the range 0.8 " to 1.2 ". While this is not exceptional, we find this range of image quality has little effect on our measured quantities. As we discuss, these 'red' QSOs have obscured nuclei, so that nuclear light is not dominant, and the host galaxies are large enough to be resolved. The lower panels in Figure 1 show the ranges of data properties that may affect the results: the dynamic range and limiting surface brightness depend on image quality, sky brightness, and the nuclear and host signals. As we note below, we measure host galaxies to an average radius of 8 arcsec, where very low surface brightness can be detected.

The objects imaged were chosen to fit the time of observation, without regard (or knowledge) of any property other than position in the sky. Data on about 90 objects were

received, but some of these were obtained in poor conditions, and we discarded them. Our 
final set of objects with good data totals 76. Figures 2 and 3 show how these are distributed in redshift, magnitude, and nuclear spectral classification (as described in the introduction). We feel we have a good representative sample of the 2MASS low redshift AGN, in redshift, apparent magnitude, nuclear spectral classification, and also radio flux.

Table 1 lists the QSOs and their properties. The nuclear classification and magnitudes are from the 2MASS database, and we have added a column giving the radio power based on positional coincidence to within 2" in the NVSS and FIRST radio surveys. Other properties listed are from the new CFHT data.

\section{Measurements}

The QSOs are all imaged in the centre of one of the $2 \mathrm{~K} \times 4 \mathrm{~K}$ CCDs in the camera, and all were identified unambiguously by comparison with the digitised sky survey images. Provided the two images of each field had comparable signal levels, sky brightness, and image FWHM, they were combined, with CR-rejection, before making further measurements. In a few cases only one image was useful.

The image processing was done using the tasks in IRAF/STSDAS. Several suitable stars were chosen to establish the PSF for each image. A PSF was generated by shifting and adding the images of the selected PSF stars. These had to be within a few arcmin of the QSO, to have similar signal levels, and to be free of blemishes or close companions.

In most cases, the host galaxies are well-resolved down to small radii (a few pixels), so that modelling and removing the PSF is not a critical step in the image analysis. However, in all cases, we generated and measured a set of PSF-subtracted images, using a range of PSF scale factors, and a few different positional shifts. The range of scale factors and PSF positions in each case was confined to those leaving a rising signal to the host galaxy 
nucleus, and the range of positional shifts until the central pixels showed a clear S-wave result due to misaligned peaks.

Using best-fit ellipses (the IRAF task 'ellipse'), azimuthally averaged luminosity plots were generated from each PSF-subtracted image, and plotted as magnitudes against both radius and $\mathrm{R}^{1 / 4}$. These plots were used to choose the best fits to exponential or de Vaucouleurs law profiles, and hence derive the associated host galaxy signal. This standard process is straightforward and will clearly distinguish disk and spheroid type galaxies which are well-resolved and have no asymmetries or superposed companions in the line of sight. In a number of cases, we found this to be so. However, in most cases, there are asymmetries or nearby objects and there is no simple host morphology, or quantitative way to separate the nuclear and host galaxy fluxes. Figure 4 shows some examples of luminosity profiles, and model fits to the PSF-subtracted images.

In cases where the QSO images have nearby companions or bright knots that have no clear connection with the host galaxy, they were edited from the image before doing the PSF-subtraction and profile-fitting, in order to look for an exponential or de Vaucouleurs profile fit to the outer host galaxy. (In our final assessment of the host galaxies, the presence of these features in the original image was taken into account.) The overall host galaxy 'type' was quantified by measuring the best linear fit to the two types of plot. A clearly exponential profile is assigned a value 1.0, while a clearly spheroidal profile has value 2.0. In a number of cases, the profile was clearly one or the other, but had regions of poor fit. These were regarded as 'disturbed' disks or spheroids, and given values between 1 and 2, depending on the length of the profile lying outside the linear fit. A number of plots had different regions of good fit to the different profiles, (usually resembling an inner bulge and outer disk structure), and these were given values of 1.3 to 1.7 , depending on the relative lengths of the two regions of linear fit in the profiles. Finally, there were many host galaxies 
that are very irregular or asymmetrical, and show no regions of linear fits in any plots. Usually, these are clearly merging or tidally disturbed galaxies, and they are given host type values of 3.0 .

The ellipse task generates formal errors on the signal values for each 'radius' (semimajor axis values), which become larger as the outer profiles disappear into the image sky noise. The sky value was determined as the median value from 1-2 arcmin around the QSO. However, the presence of faint background galaxies makes this uncertain over a small range, and we adjusted the sky value by small amounts to maximise the radial extent of the linear fits through the well-determined inner regions of the host galaxies. This exercise allowed us to note the lowest signal value that was not affected by uncertainties in the sky value. This lowest signal was noted in each case, and used to determine the dynamic range of each image, and the limiting surface brightness for each object. We also noted the eccentricity of the fitted ellipse to the isophote at this lowest level, as a measure of the overall circularity of the host galaxy.

The photometric calibration was derived from standard star observations taken as part of the service observing. All signals were converted to magnitudes, and the calibration is expected to be good to within 0.1mag. The B and $\mathrm{R}$ magnitudes listed are from the USNOA-2.0 catalogue, which has systematic errors for extended objects, as it uses a diameter-magnitude calibration for stars. Our R-band magnitudes are fainter than these by an average that runs from 0.3 at (our) magnitude 18, to 1.4 at magnitude 15 . The B-R values are likely to be about right, since the USNOA systematic errors will cancel. We consulted the CFHT 'skyprobe' monitor data for the times of our observations and noted those where 'variable absorption' was measured. With the exception of a few objects, the plot of our magnitudes against those from the USNOA have the same scatter $( \pm 0.3 \mathrm{~m})$ for good and variable nights. We thus adopt this value as the presumed accuracy of 
our magnitudes. Where we have separated the nuclear and host galaxy magnitudes, the differences (or nuclear/host ratios) do not depend on the photometric accuracy of the images.

For each best-fit profile model, we looked at the range of PSF scale factors within which the fit does not change significantly (in terms of residuals from linear fits). This yields an error bar for the host galaxy flux, and hence the ratio of nuclear to host flux (hereafter $\mathrm{N} / \mathrm{H}$ ). Another estimate of robustness of host galaxy fluxes is comparison of the values for best-fit exponential and spheroidal models, to see how model-dependent the numbers are. In all cases, a best overall gradient for an exponential fit was recorded, to provide a rough number describing the scale length of the host galaxy structure.

In general, the host galaxies were detected to radii of 40-50 pixels, or 8-10 arcsec. Thus, they are all large and well-resolved, even with the poorest image quality in the dataset (see Figures 1 and 4). At these radii, we reach limiting surface brightness values near 27 magnitudes per square arcsecond in many cases. Our host galaxy fluxes are estimated to be correct to within 0.3 magnitudes for all objects selected for this paper.

Three of the QSOs have high N/H ratios, more like those typical of blue-selected QSOs. The host galaxy properties are less reliable for these. We discuss them in more detail below.

For all host galaxies, we have assigned an 'interaction level' index from 0 to 3 . This is an attempt to quantify merging and tidal disturbances that have been associated with triggering of nuclear activity in many types of AGN. Level 3 indicates large-scale and clear tidal disturbance, while level 0 indicates a symmetrical and undisturbed morphology. Other indicators in between include very asymmetrical arms, knots with connecting luminous bridges to the main galaxy, an off-centred nucleus, outer arcs, a warped disk, or nuclear jet-like features. This index was estimated independently by the two principal authors, with a high degree of agreement. Figures 5 and 6 illustrate some examples of interaction 
levels 1 through 3 . In three cases, the merging event is so extreme that we did not attempt to measure the host and nuclear magnitudes, or fit ellipses to the images.

Table 1 shows all the measured quantities for the 76 QSOs in the sample. In addition to the quantities discussed above, we have given absolute $\mathrm{R}$ magnitudes for the whole object, and the host galaxy for the different fitted models. These are based on $\mathrm{H}_{0}=70$, and no k-corrections have been applied.

\section{Selection effects}

We expect some selection effects in a flux-limited sample spanning a redshift range of a factor more than 4 . We expect that the higher redshift host galaxies will be less well resolved: however, the plot of $\mathrm{N} / \mathrm{H}$ flux ratio against redshift shows no real trend (see Figure 7). The least-resolved objects are not at the highest redshift, and plots of $\mathrm{N} / \mathrm{H}$ against image FWHM or the limiting signal show that these quantites dominate over redshift in determining the resolved fraction. Thus, the random mix of image quality removes almost all of the redshift bias in the resolved fraction of the total QSO light. We also note that the least resolved QSOs have good to average image FWHM, so the measurements do not appear to be biased by image quality either.

The host galaxy scale length in arcsec generally decreases with increasing redshift, and the scale lengths in rest-frame Kpc are similar at all redshifts in our range. The host galaxy contour ellipticities have an upper envelope decreasing with increasing redshift (see Figure 7). This may be real (more distant hosts are more circular), or perhaps that we are seeing faint irregular outer structure in some nearby objects, and do not reach faint enough to see this at higher redshifts.

The mean and spread of redshift where we see the different interaction levels are very 
similar, so that we see no redshift bias in this quantity. We do see a systematic increase in mean redshift, moving from disk to mixed to spheroid morphology hosts. The irregular morphology hosts have a similar redshift distribution and average to the pure disk systems. The spheroid systems house more luminous QSOs, so that the connection with redshift is probably real, and is discussed below.

We also looked for trends connecting measured quantities with data quality values (FWHM, dynamic range, limiting surface brightness, sky brightness). None were found.

\section{Null effects}

Having found no significant sample or data-quality biases in the sample, we looked for trends and correlations among the QSO properties and our measured quantities. In the next section we discuss those that may be significant. However, it is also of interest to note where no correlation is seen, particularly where they might test a scenario or process.

The ellipticity of the host galaxies might be expected to correlate with the interaction level index, since interactions generally lead to strong deviations from circular symmetry. There is a wide range of overlap for all interaction levels, and while the mean ellipticity is lowest for the non-interacting de Vaucouleur-law hosts, and largest for the level 3 objects, the differences are not significant. This lack of significant correlation is presumably because the ellipticity is mainly a projection effect for any type of galaxy. Similarly, the ellipticity shows little trend with nuclear type: the type 1 objects have lower e values overall, possibly indicating they occur more in face-on disks. We also find no trends of the luminosity scale lengths with any other quantities.

Host morphology index has the same mean colour for all, again with large spread. The J-K/B-R plot shows no correlation or grouping, so the overall SEDs are presumably a 
complex mix of stars and dust.

Looking at the radio fluxes, we find no correlation with $\mathrm{N} / \mathrm{H}$ ratio, nuclear spectral type, or host galaxy interaction level.

\section{Trends and correlations}

Our most important measured quantities are the fluxes and morphological classifications of the host galaxies. These are measured in various ways, and summarized in Table 1.

The overall ratio of nuclear flux to host galaxy flux is the simplest quantity, and the variation of this quantity with nuclear spectral classification is shown in Figure 8. The $\mathrm{N} / \mathrm{H}$ flux ratio is highest for type $1 \mathrm{~s}$, and lowest for LINERs. This is in accordance with the orientation-bias assumed to explain the type 1-2 differences, and the lower nuclear luminosity of LINERs. Thus, the 2MASS sample seems normal in this respect. However, compared with 'standard' QSOs in this redshift range, the actual values of N/H are lower by several times, suggesting that the entire nuclear regions are surrounded by dust. The mean N/H from a sample of normal QSOs in this redshift range by Hutchings and Neff (1991) has a mean of 5 , compared with a mean of 0.5 for the 2MASS sample. The overall absolute magnitudes show a similar result: a decline of 0.7 magnitudes from nuclear types 1 to 3, which is almost all in the nuclear flux - the host galaxy absolute magnitudes show little change along the sequence.

The $\mathrm{N} / \mathrm{H}$ ratio also varies with interaction level index. Non-interacting hosts have the highest $\mathrm{N} / \mathrm{H}$ ratio, but the highly interacting hosts have very similar values. The intermediate interaction level hosts show the lowest mean values and upper values, as shown in Figure 8. The $\mathrm{N} / \mathrm{H}$ ratio also varies with overall colour (which is presumably linked to the interaction index). The sequence with interaction index is interesting: non-interacting 
and index value 2 objects have blue colours (both $B-R=0.8$ ) while the strongest interaction objects have $\mathrm{B}-\mathrm{R}=1.2$ and the mildest interactions are reddest at $\mathrm{B}-\mathrm{R}=1.6$. As the colours refer to host plus nucleus, this can be explained by sequences of nuclear dust-formation and clearing, and star-formation in more extended regions that are less dust-covered. We discuss this further in the next section. The envelope of $\mathrm{B}-\mathrm{R}$ with $\mathrm{N} / \mathrm{H}$ ratio shows that high ratios occur for the bluest colours, as we would expect for dust extinction as the cause of the red colour (Figure 8).

The QSO+host absolute magnitudes average at -22.5 in $\mathrm{R}$, which is not particularly luminous. However, if we assume there is an average 2.5 magnitudes of extinction (by the comparison with blue-selected QSOs), the objects have absolute magnitudes averaging -25: i.e. fairly luminous QSOs with significant dust extinction. The distribution of N/H flux shows a clear boundary with B-R colour, also showing that the highest ratios are seen in the bluest objects in the sample. We attempted to make a correction for nuclear reddening based on overall B-R, and using our measured nucleus/host ratios, obtained estimates of the nuclear absolute magnitudes. These range from -19 to -27 , with upper limit values dropping through nuclear types 1 through 3, as expected. However, as the colours are for the combined host and nucleus, this estimate has large uncertainties in individual objects. It is probably good for the average: nuclear $\mathrm{M}_{R}-23.5$ for type $1,-22.5$ for types 2 , and -21 for the LINERS. There is no correlation between host and the corrected nuclear absolute magnitudes. The host absolute magnitudes are more reliable as they generally dominate the overall flux, and are probably not heavily reddened outside the nucleus.

The host absolute magnitudes show a trend with nuclear type, as seen in Figure 9. These range over 0.7 magnitudes, but should be compared with a matched sample of AGN not selected by NIR properties. There is no correlation of host absolute magnitude with host morphological type, although the most luminous hosts are spheroidal. The 
host morphological type shows some correlation with radio power (see Figure 9), in which the spheroid-dominated galaxies are more radio-luminous by an order of magnitude than the disk systems. This follows the general wisdom that luminous radio sources reside in

elliptical galaxies, although the radio luminosities here are not particularly high. The radio luminosities do not show any correlation with host or nuclear absolute magnitude, or B-R. Plots with K-band absolute magnitudes show very similar trends to those derived from our measured $\mathrm{R}$ band magnitudes, so are presumably not due to dust reddening effects.

\section{Discussion}

Our database provides a good measure of the properties of low redshift NIR-selected AGN. The investigation is primarily aimed at understanding whether this population is significantly different from the traditionally-selected AGN, based on blue colours and bright optical nuclei, or simply a dust-obscured subgroup of the general AGN population. While comparisons among AGN samples are always plagued by selection effects, we may make a few comparisons, and note the unusual properties of our sample, in addressing this issue.

In terms of known comparable objects from other catalogues, we note the following.

Excluding LINERS (only 13\% of the 2MASS sample), in the redshift and declination range studied, the 2MASS sample has 230 objects, the Hewitt-Burbidge (1993) catalogue 364, and the Veron-Veron (1998) catalogue 310. As noted in the introduction, there is no overlap between 2MASS and previously catalogued AGN. Thus, the 2MASS sample represents a major new population of low redshift AGN, comparable with the traditionally selected objects. Since spectroscopic verification of other candidates is incomplete, the population of red AGN is likely to be signficantly higher, and will be the subject of a separate paper.

The mean B-R colours are 1.1 for the 2MASS objects, compared with the (not 
well-established) value near 0.3 for standard AGN. Our N/H ratios average at 0.5 compared with 5.0 for standard AGN. These numbers indicate that the 2MASS AGN have nuclear extinction of an average factor close to 10 compared with standard AGN, if they are basically the same objects. The distribution of B-R falls rapidly above 1.5 so that it appears unlikely that there is a large population of even more dust-hidden AGN, obscured even at 2 microns.

The three QSOs with high N/H flux ratios (more typical of standard QSOs) are probably not typical of the 2MASS AGN. Two of them have very blue colours, and the third is very bright, where the catalogue magnitudes are least reliable (indeed our R magnitude suggests a bluer colour). One of them appears in standard QSO catalogues. We have ignored them in the overall discussion, although they are pointed out in some of the diagrams.

The host galaxies show a high degree of tidal interaction. 30\% of the sample is highly interacting, and $71 \%$ have some sign of interaction. The numbers for standard AGN are not easy to compare because of the different data quality and samples in the literature. The sample of Hutchings and Neff (1992) of 28 objects, almost all of z less than 0.32, was taken with better optical resolution, and was able to detect more subtle signatures of interaction than we can in this paper. However, only 3 of these show marked signs of interaction, and another 8 moderate signs that we would find in the 2MASS sample data. However, they do find signs of old mergers and mergers with high mass ratios (13 and 10 in their sample, 5 in both classes). Thus, a comparison with low z QSOs (type 1 objects) indicates fractions of $11 \%$ and $39 \%$ in standard QSOs to compare with the $33 \%$ and $75 \%$ in the type 1 objects in the 2MASS sample.

Another comparison of interest is with IRAS-selected galaxies, not selected as housing AGN. A sample of 64 of these was investigated, also with the CFHT, by Hutchings and 
Neff (1991), and 43 of them do host AGN, from QSOs to LINERs. These are at much lower redshift (average $\mathrm{z} \sim 0.06$ ), but many of these galaxies are in major mergers, which would be very obvious even in blue QSO hosts at larger redshifts. The sample of 43 IRAS AGN has mean interaction strength and age in the middle of the range, with some $23 \%$ being weak or old interactions. These objects are more similar to the 2MASS AGN, than the traditionally-selected AGN, in terms of their interaction status.

A final comparison of interest is with an X-ray selected one, which may be free of the optical-IR biases (but may embody other biases such as orientation, and hard X-ray sensitivity). Schreier et al (2001) and Koekemoer et al (2002) discuss HST imaging of X-ray sources in the HDF-S field. Their study notes that the optically bright sources are red and consistent with dust obscured nuclei in merging galaxies, but at higher redshifts than the 2MASS sample. They also find a fainter population they speculate are type 2 AGN at much higher redshift. It is possible that the red QSO population dies beyond redshift 0.3. Deeper imaging of a larger sample would help expand this comparison.

In our profile-based classifications, only 27 of the sample of $76(36 \%)$ clearly fit a simple standard galaxy model, about evenly split between disk and spheroid (12 and 15). Thus, the great majority of the 2MASS host galaxies are disturbed, by any measure.

It has been widely discussed (see e.g. Sanders and Mirabel 1996 and references therein, Hutchings and Neff 1991) that mergers often lead to accumulations of gas in the central regions of the galaxy, which triggers circumnuclear star-formation and possibly activates the central black hole(s). The circumnuclear starburst generates large amounts of dust, that obscure the central hot stars and AGN, and re-radiate in the IR. As the starburst dies down, the central dust clears, revealing the AGN, which continues to be fuelled until many of the initial events of the merger have died away. Many standard AGN are found in weakly interacting systems, where the initial mass ratio may be a few, rather than the massive 
mergers of equals we see in the IR-selected objects, again suggesting that the AGN fuelling is relatively long-lived and steady compared with the tidal disturbances and accompanying starbursts.

Thus, the 2MASS sample appears to bridge the gap between massive young mergers and minor mergers that do not produce massive starbursts and dust, while still fuelling an AGN. Given the low overlap with standard QSOs, it appears that these red QSOs are a significant population, at least at low redshift. There seems to be no reason to suppose they do not exist in higher redshift populations in the similar proportions.

The overall colour changes with our interaction index must indicate that age and strength of the interaction, and accompanying star-formation and nuclear extinction, all play a role in the overall colour. We note in this connection that intermediate interactions have relatively blue colours and lower luminosity AGN.

The host galaxy luminosities and other properties are typical of standard AGN, so that these objects do not appear to arise in a very different host galaxy population, or to have unusual nuclear properties.

There is a related study of 2MASS QSOs by Marble et al (2003), which uses HST snapshot images of 29 objects, of which 7 have redshifts above 0.3 , which was done independently from ours. These images are measurable only to radii of $2-3$ arcsec in most cases, and thus do not reveal the faint traces of interactions of the CFHT data. With this caveat, their results are in agreement with this paper.

\section{Summary}

Our investigation of the host galaxies of 2MASS-selected red AGN has the following principal conclusions. 
1. The host galaxies have a $2-3$ times higher fraction of obvious tidal interactions than matched samples of traditionally-selected AGN at the same redshift.

2. The luminosity profiles show that most $(64 \%)$ of the host galaxies are not fit by a simple galaxy model.

3. The $\mathrm{N} / \mathrm{H}$ ratio is 10 times lower than for traditionally-selected QSOs. This, and the average colour, indicates dust extinction of normally luminous nuclei.

4. The host galaxy properties are not otherwise unusual and do not suggest a different parent population.

5. Red QSOs form a major, previously unknown population of low-redshift AGN. The details of their nuclear triggering and fuelling may be different from traditionally-selected QSOs.

We thank the CFHT service observers, and Ann Gower for discussions and support of N. Maddox. 


\section{References}

Cutri R.M., Nelson B.O., Kirkpatrick J.D., Skrutskie M.F., Huchra J.P. (in preparation)

Hewitt A., and Burbidge G.R., 1993, ApJS, 87, 451

Hutchings J.B. and Neff S.G., 1991, AJ, 101, 434

Hutchings J.B. and Neff S.G., 1992, AJ, 104, 1

Hutchings J.B., Janson T., and Neff S.G., 1989 ApJ, 342, 660

Koekemoer A.M., et al 2002, ApJ, 567, 657

Marble A.R., et al, 2003, ApJ (in press), astro-ph 0303184

Sanders D.B., and Mirabel I.F. 1996, Annu Rev Astron Astrophys 34, 749

Schreier E.J. at al, 2001, ApJ, 560, 127

Veron-Cetty M-P., and Veron P., 1998, ESO scientific report \#18

Warren S.J., Hewett P.C., Foltz C.B., 2000, MNRAS, 312, 827 


\section{Captions to figures}

1. Distributions of data properties in sample. The dynamic range is from QSO nucleus to lowest contour used, and the limiting surface brightness measures this lower level.

2. Photometric properties of the observed sample (circled points) compared with entire 2MASS sample. The observed objects were selected to fit the service observing schedule and are well spread over the full sample. The sample is limited by $\mathrm{K}$ magnitude.

3. Distributions in redshift of the three nuclear spectral classifications. Apart from the lowest redshift objects (all class 1), the distributions sample the redshift range comparably. The dots indicate objects with known radio flux. These too are well distributed.

4. Illustrative luminosity profiles from the sample. The lines show the raw QSO and PSF, scaled to the same peak value. The dots and dashed lines are the best-fit PSF-subtracted profile, for exponential (left) and $\mathrm{R}^{1 / 4}$ models (right). The QSO names are truncations of the positions given in Table 1. 0411-01 is very irregular and does not fit either model. 1507-12 can be fit reasonably well by either model, while 0215-15 and 1221-11 fit only one model well.

5. Examples of highly interacting host galaxies. Clockwise from top left, the QSOs are 0411-01 (foreground star removed for profile fitting), 1001-05, 1521-11, 1006+07.

6. Examples of less interacting host galaxies: index 2 (top) and index 1 (bottom). Clockwise from top left: $1517+17$ (highly asymmetrical galaxy with tidal arm); 1519+18 (double nucleus and asymmetrical halo); 1517-06; and 2124-17 (both lower ones have asymmetrical outer arms and shells).

7. Measured quantities as function of target redshift. Upper: the resolved fraction shows no change with z, and depends more on image resolution (see text). Lower: the outer host galaxy ellipticity may decrease with increasing redshift, as outer host galaxy structures 
become smaller and fainter.

8. Distributions of properties with ratio of nuclear to host $(\mathrm{N} / \mathrm{H})$ flux. Arrows indicate two extreme values which are off-scale. The dashed lines connect mean values (without the two extreme objects). Note the progression of nuclear dominance from type 1 to 2 to LINERS, and the lower nuclear dominance in mid-range interacting hosts. The upper envelope of B-R with nuclear dominance suggests that nuclear dust-obscuration is a major effect in the sample.

9. Upper: distributions of host absolute magnitude with nuclear spectral type. Note the progression in mean values from types 1 to 2 to LINERS, and the larger range in type 1 objects. Lower: radio power with host morphology type. Note the trend of higher radio power in the sequence from disk-dominated (type 1) to spheroid-dominated (type 2) host galaxy. Irregular (interacting) hosts fit the value for disturbed disks. Dashed lines connect the mean values for the groups. 
Table 1: 2MASS sample and measurements

\begin{tabular}{|c|c|c|c|c|c|c|c|c|c|c|c|c|c|c|c|}
\hline $\begin{array}{r}\text { RA } \\
(2\end{array}$ & Dec & $\begin{array}{l}\text { Nuc } \\
\text { type }\end{array}$ & $\mathrm{z}$ & $\begin{array}{c}\mathrm{J}, \mathrm{K}, \mathrm{B}, \mathrm{R} \\
\text { (catalogue) }\end{array}$ & $\begin{array}{c}\text { fwhm } \\
\text { (") }\end{array}$ & $\begin{array}{l}\text { QSO,host } \\
\text { (mag) }\end{array}$ & $\begin{array}{l}\text { host } \\
\text { type }\end{array}$ & $\begin{array}{l}\text { range } \\
\text { (mag) }\end{array}$ & $\begin{array}{l}\text { limit } \\
\mathrm{m} / " 2\end{array}$ & $\mathrm{e}$ & $\begin{array}{l}\mathrm{M}_{r} \\
\mathrm{QSO}\end{array}$ & $\begin{array}{c}\text { Int } \\
\text { index }\end{array}$ & $\mathrm{n} / \mathrm{h}$ & $\begin{array}{c}\text { scale } \\
(\mathrm{m} / \mathrm{kpc})\end{array}$ & $\begin{array}{l}20 \mathrm{~cm} \\
\mathrm{WHz}\end{array}$ \\
\hline $2: 15: 38.40$ & $-15: 10: 12.4$ & 1 & 0.211 & $14.6,12.4,15.4,15.3$ & 1.3 & $15.5,16.1$ & 2 & 10.4 & 27.0 & 0.1 & -24.3 & 0 & 0.70 & 0.2 & - \\
\hline $2: 21: 50.60$ & $13: 27: 41.0$ & 2 & 0.140 & $15.6,13.2,18.9,16.4$ & 1.5 & $16.6,16.7$ & 3 & 6.8 & 26.5 & 0.4 & -22.3 & 3 & 0.06 & 0.3 & 22.7 \\
\hline $2: 26: 50.25$ & $13: 43: 38.7$ & 1 & 0.194 & $15.8,13.8,19.9,17.0$ & 1.4 & $17.5,17.7$ & 1 & 5.6 & 25.1 & 0.3 & -22.2 & 1 & 0.24 & 0.3 & - \\
\hline $2: 31: 01.74$ & $14: 26: 24.2$ & 1 & 0.259 & $16.2,14.0,19.6,18.0$ & 1.4 & $17.8,18.1$ & 1.7 & 7.5 & 27.4 & 0.2 & -22.6 & 1 & 0.29 & 0.2 & - \\
\hline $2: 48: 07.37$ & $14: 59: 57.7$ & 1 & 0.072 & $14.8,12.7,14.5,13.1$ & 2.0 & $15.2,15.3$ & 1 & 7.3 & 25.8 & 0.0 & -22.1 & 2 & 0.15 & 0.3 & - \\
\hline $3: 06: 52.43$ & $-5: 31: 56.4$ & 1 & 0.126 & $15.2,12.9,16.6,15.9$ & 2.5 & $16.3,16.5$ & 3 & 5.9 & 25.5 & 0.2 & -22.4 & 1 & 0.21 & 0.3 & - \\
\hline $3: 12: 31.03$ & $7: 06: 55.0$ & 1 & 0.145 & $15.5,13.4,18.1,16.0$ & 2.4 & $16.9,17.1$ & 1 & 5.5 & 25.6 & 0.5 & -22.1 & 1 & 0.18 & 0.2 & - \\
\hline $3: 13: 02.25$ & $21: 07: 14.5$ & 3 & 0.094 & $15.8,13.8,17.9,16.9$ & 1.0 & $17.0,17.1$ & 1.3 & 5.2 & 25.3 & 0.3 & -20.7 & 3 & 0.03 & 0.4 & 22.9 \\
\hline $4: 00: 19.77$ & $5: 02: 14.6$ & 2 & 0.187 & $14.8,12.7,17.0,16.0$ & 1.0 & $17.8,18.3$ & 1.5 & 8.3 & 27.0 & 0.2 & -21.9 & 3 & 0.54 & 0.2 & 23.5 \\
\hline $4: 09: 24.86$ & $7: 58: 56.1$ & 1 & 0.091 & $14.9,12.7,17.4,16.2$ & 1.2 & $16.5,17.0$ & 2 & 9.1 & 26.7 & 0.3 & -21.3 & 3 & 0.61 & 0.4 & - \\
\hline $4: 11: 26.47$ & $-1: 18: 05.6$ & 3 & 0.139 & $16.7,14.5,18.2,16.2$ & 0.8 & $17.8,17.9$ & 3 & 6.3 & 25.7 & 0.5 & -21.1 & 3 & 0.14 & 0.3 & - \\
\hline $4: 22: 56.57$ & $-18: 54: 42.1$ & 1 & 0.064 & $13.8,11.6,13.6,13.7$ & 1.4 & $15.3,15.7$ & 1.5 & 9.9 & 27.0 & 0.2 & -22.0 & 2 & 0.47 & 0.7 & 22.1 \\
\hline $4: 35: 22.56$ & $-6: 35: 26.1$ & 1 & 0.185 & $15.3,13.3,16.8,16.1$ & 1.0 & $16.5,17.0$ & 1.3 & 7.9 & 25.3 & 0.3 & -23.1 & 3 & 0.49 & 0.2 & - \\
\hline $4: 36: 48.40$ & $-11: 23: 55.9$ & 1 & 0.208 & $15.9,13.7,16.5,16.2$ & 1.2 & $17.3,17.7$ & 2 & 7.5 & 25.9 & 0.1 & -22.5 & 0 & 0.50 & 0.3 & - \\
\hline $4: 47: 47.62$ & $-16: 49: 34.7$ & 1 & 0.199 & $15.8,13.7,18.0,16.1$ & 1.0 & $17.3,17.5$ & 1.5 & 7.4 & 26.5 & 0.0 & -22.3 & 3 & 0.14 & 0.3 & - \\
\hline $5: 04: 25.68$ & $-19: 09: 25.4$ & 2 & 0.138 & $15.6,13.5,16.4,15.9$ & 1.1 & $16.9,17.1$ & 1.5 & 6.5 & 25.6 & 0.3 & -22.0 & 1 & 0.16 & 0.3 & 23.7 \\
\hline $8: 16: 52.26$ & $42: 58: 29.4$ & 1 & 0.235 & $15.8,13.7,17.3,17.5$ & 1.0 & $16.9,20.0$ & 1.5 & 9.2 & 26.3 & 0.1 & -23.3 & 0 & 17.0 & 0.1 & - \\
\hline $8: 19: 09.03$ & $34: 19: 31.3$ & 3 & 0.228 & $16.6,14.6,19.5,18.0$ & 0.9 & $18.1,18.2$ & 3 & 7.0 & 27.0 & 0.5 & -22.0 & 3 & 0.10 & 0.2 & - \\
\hline $8: 56: 32.98$ & $59: 57: 46.7$ & 1 & 0.283 & $16.0,14.0,16.7,16.5$ & 1.2 & $16.5,19.3$ & 1.2 & 6.0 & 27.4 & 0.1 & -24.1 & 2 & 12.2 & 0.2 & - \\
\hline $8: 59: 21.28$ & $59: 48: 20.7$ & 2 & 0.280 & $16.4,13.8,19.2,17.8$ & 1.2 & $18.3,18.5$ & 1.8 & 7.0 & 27.5 & 0.1 & -22.3 & 2 & 0.20 & 0.2 & - \\
\hline 9:01:51.18 & $34: 57: 24.1$ & 1 & 0.274 & $16.3,14.2,18.1,17.1$ & 1.1 & $17.5,18.0$ & 1.5 & 7.1 & 25.8 & 0.2 & -23.0 & 0 & 0.60 & 0.3 & - \\
\hline $9: 08: 37.68$ & $34: 24: 54.2$ & 3 & 0.202 & $16.1,13.9,19.5,17.7$ & 0.9 & $18.1,18.2$ & 1.5 & 7.3 & 27.0 & 0.3 & -21.6 & 1 & 0.14 & 0.4 & - \\
\hline $9: 10: 00.75$ & $33: 48: 09.2$ & 2 & 0.178 & $16.6,14.3,18.7,17.7$ & 1.3 & $17.8,18.0$ & 1.5 & 7.6 & 27.0 & 0.1 & -21.7 & 0 & 0.22 & 0.5 & 23.1 \\
\hline $10: 01: 18.15$ & $41: 04: 13.2$ & 3 & 0.143 & $16.2,14.2,16.3,15.7$ & 1.2 & $16.9,-$ & 3 & - & - & - & -22.0 & 3 & - & - & 22.8 \\
\hline 10:01:39.17 & $-5: 58: 13.2$ & 1 & 0.217 & $15.8,13.6,16.4,16.7$ & 1.1 & $16.9,17.3$ & 3 & 8.8 & 26.7 & 0.1 & -23.1 & 3 & 0.50 & 0.3 & - \\
\hline 10:04:11.76 & $30: 10: 32.1$ & 3 & 0.257 & $16.7,14.7,19.0,18.4$ & 1.1 & $17.7,17.9$ & 1.3 & 7.5 & 27.0 & 0.0 & -22.7 & 2 & 0.15 & 0.1 & 23.4 \\
\hline 10:06:02.49 & $7: 11: 32.3$ & 2 & 0.120 & $15.3,13.2,14.8,13.3$ & 0.9 & $14.79,-$ & 1.3 & - & - & - & - & 3 & - & - & 23.0 \\
\hline $10: 06: 57.84$ & $41: 04: 06.6$ & 1 & 0.089 & $15.8,13.8,16.9,15.9$ & 1.0 & $16.5,16.9$ & 3 & 8.0 & 25.7 & 0.2 & -21.2 & 3 & 0.40 & 0.4 & - \\
\hline $10: 10: 34.28$ & $37: 25: 14.8$ & 1 & 0.283 & $16.1,13.8,19.0,17.7$ & 1.0 & $17.9,18.4$ & 2 & 8.3 & 26.7 & 0.3 & -22.6 & 1 & 0.53 & 0.2 & - \\
\hline $10: 13: 28.87$ & $32: 40: 10.2$ & 1 & 0.287 & $16.8,14.7,17.9,17.5$ & 1.1 & $18.0,18.4$ & 1.5 & 7.7 & 27.0 & 0.1 & -22.6 & 1 & 0.38 & 0.3 & - \\
\hline $10: 14: 00.48$ & $19: 46: 14.4$ & 1 & 0.110 & $14.4,12.4,16.5,14.0$ & 1.1 & $15.6,-$ & 3 & - & - & - & -22.7 & 3 & - & - & 23.9 \\
\hline 10:14:05.89 & $0: 06: 20.5$ & 1 & 0.141 & $15.3,13.2,18.2,16.3$ & 1.1 & $16.5,16.8$ & 2 & 8.2 & 26.3 & 0.2 & -22.4 & 1 & 0.24 & 0.2 & - \\
\hline $10: 14: 21.19$ & $20: 10: 32.4$ & 1 & 0.261 & $17.0,14.7,17.8,17.9$ & 0.9 & $17.9,18.4$ & 1.5 & 9.1 & 27.5 & 0.1 & -22.5 & 0 & 0.60 & 0.5 & - \\
\hline $10: 40: 43.66$ & $59: 34: 09.2$ & 2 & 0.148 & $14.8,11.8,18.4,17.5$ & 1.3 & $17.9,18.1$ & 2 & 7.5 & 26.7 & 0.3 & -21.2 & 0 & 0.20 & 0.4 & 23.3 \\
\hline $10: 57: 28.63$ & $-13: 53: 59.5$ & 3 & 0.163 & $16.1,13.6,18.7,17.3$ & 1.1 & $17.7,17.9$ & 1.4 & 7.5 & 26.1 & 0.1 & -21.6 & 0 & 0.20 & 0.4 & - \\
\hline $11: 06: 26.66$ & $-13: 13: 06.9$ & 1 & 0.286 & $15.9,13.7,16.4,16.4$ & 0.9 & $17.4,17.8$ & 2 & 8.5 & 24.5 & 0.2 & -23.2 & 0 & 0.45 & 0.2 & - \\
\hline $11: 09: 40.52$ & $-18: 43: 13.6$ & 1 & 0.214 & $15.9,13.7,16.2,15.8$ & 1.2 & $17.2,17.3$ & 1.5 & 9.0 & 26.3 & 0.4 & -22.7 & 2 & 0.10 & 0.2 & - \\
\hline $11: 10: 09.77$ & 13:58:06.1 & 1 & 0.215 & $16.5,14.5,18.4,18.0$ & 1.2 & $17.8,18.0$ & 1.5 & 7.6 & 26.7 & 0.0 & -22.2 & 0 & 0.27 & 0.4 & - \\
\hline $11: 12: 35.74$ & $13: 54: 50.6$ & 3 & 0.236 & $16.5,14.4,18.8,17.6$ & 1.2 & $17.9,18.2$ & 2 & 5.0 & 24.4 & 0.2 & -22.3 & 3 & 0.36 & 0.4 & - \\
\hline $11: 16: 03.16$ & $2: 08: 52.5$ & 1 & 0.211 & $15.2,13.1,16.4,16.0$ & 1.3 & $16.4,17.4$ & 3 & 7.6 & 25.3 & 0.3 & -23.4 & 3 & 1.50 & 0.2 & 22.4 \\
\hline $11: 27: 51.14$ & $24: 32: 08.1$ & 1 & 0.088 & $15.0,12.9,17.9,15.9$ & 1.1 & $16.4,16.7$ & 1 & 6.8 & 25.1 & 0.6 & -21.4 & 0 & 0.32 & 0.3 & - \\
\hline $11: 31: 11.05$ & $16: 27: 39.5$ & 2 & 0.174 & $16.3,14.1,18.1,16.8$ & 1.0 & $16.9,17.1$ & 3 & 6.4 & 25.3 & 0.5 & -22.5 & 3 & 0.18 & 0.1 & 22.5 \\
\hline $11: 36: 08.12$ & $18: 01: 33.7$ & 2 & 0.232 & $17.0,14.8,19.5,18.2$ & 0.9 & $18.4,18.5$ & 2 & 5.8 & 25.6 & 0.3 & -21.7 & 1 & 0.09 & 0.2 & 22.9 \\
\hline $11: 58: 24.61$ & $-30: 03: 34.9$ & 1 & 0.136 & $15.1,13.0,15.2,14.7$ & 2.4 & $16.4,16.5$ & 1 & 9.0 & 26.5 & 0.2 & -22.4 & 2 & 0.10 & 0.4 & 23.2 \\
\hline $12: 11: 15.72$ & $-14: 46: 21.5$ & 1 & 0.218 & $15.5,13.4,17.7,16.8$ & 0.8 & $17.1,17.6$ & 2 & 8.0 & 26.1 & 0.2 & -22.9 & 0 & 0.58 & 0.2 & - \\
\hline $12: 12: 14.49$ & $-14: 22: 16.1$ & 1 & 0.148 & $15.8,13.7,18.3,17.5$ & 1.2 & $17.5,17.8$ & 1.5 & 9.0 & 27.0 & 0.4 & -21.6 & 0 & 0.28 & 0.6 & - \\
\hline $12: 21: 25.33$ & $-11: 11: 37.7$ & 2 & 0.209 & $14.9,12.7,16.9,16.4$ & 1.1 & $16.5,16.9$ & 2 & 9.0 & 26.3 & 0.1 & -23.3 & 0 & 0.45 & 0.2 & 24.7 \\
\hline 13:07:00.66 & $23: 38: 05.0$ & 1 & 0.275 & $16.8,13.4,21.0,19.5$ & 1.6 & $19.4,19.7$ & 1.5 & 5.0 & 26.2 & 0.1 & -21.1 & 2 & 0.40 & 0.4 & 23.3 \\
\hline 13:09:05.12 & $4: 33: 12.2$ & 2 & 0.222 & $16.6,13.8,18.7,17.6$ & 2.0 & $18.1,18.2$ & 1.5 & 6.0 & 26.4 & 0.2 & -21.9 & 2 & 0.10 & 0.2 & 23.3 \\
\hline $13: 12: 07.64$ & $-18: 55: 51.2$ & 1 & 0.202 & $15.6,13.3,17.1,16.7$ & 1.5 & $17.5,17.7$ & 1.7 & 8.0 & 27.0 & 0.1 & -22.3 & 0 & 0.20 & 0.5 & - \\
\hline $13: 17: 04.36$ & $-17: 39: 12.6$ & 1 & 0.216 & $14.9,12.8,17.2,15.7$ & 1.2 & $17.0,17.6$ & 1.9 & 6.0 & 26.3 & 0.3 & -22.9 & 3 & 0.74 & 0.2 & - \\
\hline
\end{tabular}


Table 1, continued

\begin{tabular}{|c|c|c|c|c|c|c|c|c|c|c|c|c|c|c|c|}
\hline \multicolumn{2}{|c|}{ (2000) } & $\begin{array}{l}\text { Nuc } \\
\text { type }\end{array}$ & $\mathrm{z}$ & $\begin{array}{c}\mathrm{J}, \mathrm{K}, \mathrm{B}, \mathrm{R} \\
\text { (catalogue) }\end{array}$ & $\begin{array}{c}\text { fwhm } \\
\text { (") }\end{array}$ & $\begin{array}{l}\text { QSO,host } \\
\text { (mag) }\end{array}$ & $\begin{array}{l}\text { host } \\
\text { type }\end{array}$ & $\begin{array}{l}\text { range } \\
(\mathrm{mag})\end{array}$ & $\begin{array}{l}\text { limit } \\
\mathrm{m} / " 2\end{array}$ & $\mathrm{e}$ & $\begin{array}{l}\mathrm{M}_{r} \\
\mathrm{QSO}\end{array}$ & $\begin{array}{c}\text { Int } \\
\text { index }\end{array}$ & $\mathrm{n} / \mathrm{h}$ & $\begin{array}{c}\text { scale } \\
(\mathrm{m} / \mathrm{kpc})\end{array}$ & $\begin{array}{l}20 \mathrm{~cm} \\
\mathrm{WHz}\end{array}$ \\
\hline $13: 21: 39.08$ & $13: 42: 30.4$ & 3 & 0.199 & $17.0,14.9,20.4,18.5$ & 1.5 & $18.6,18.8$ & 1.2 & 7.0 & 27.0 & 0.1 & -21.2 & 0 & 0.20 & 0.4 & 21.8 \\
\hline $13: 23: 14.70$ & $-2: 19: 02.0$ & 1 & 0.160 & $15.0,12.8,16.9,16.2$ & 1.5 & $16.6,16.9$ & 1 & 9.0 & 27.4 & 0.2 & -22.6 & 3 & 0.32 & 0.3 & 21.6 \\
\hline $13: 29: 17.52$ & $12: 13: 40.6$ & 1 & 0.203 & $16.1,14.1,18.7,17.5$ & 1.6 & $18.9,19.4$ & 1.5 & 3.9 & 24.3 & 0.2 & -20.8 & 0 & 0.52 & 0.4 & - \\
\hline $13: 38: 45.30$ & $-4: 38: 53.0$ & 1 & 0.163 & $15.6,13.6,16.9,16.5$ & 1.5 & $16.5,17.0$ & 2 & 6.0 & 25.8 & 0.1 & -22.8 & 0 & 0.58 & 0.3 & 22.5 \\
\hline $14: 07: 37.48$ & $42: 56: 16.3$ & 2 & 0.118 & $15.4,13.1,17.2,15.5$ & 1.3 & $16.8,16.8$ & 3 & 6.7 & 25.8 & 0.3 & -21.9 & 3 & 0.13 & 0.3 & 23.0 \\
\hline $14: 17: 58.24$ & $36: 07: 41.5$ & 1 & 0.212 & $16.1,13.9,17.0,16.3$ & 1.0 & $16.6,17.0$ & 3 & 8.7 & 26.5 & 0.1 & -23.2 & 3 & 0.42 & 0.2 & - \\
\hline $14: 48: 19.38$ & $44: 32: 32.7$ & 1 & 0.080 & $14.2,12.2,16.1,13.1$ & 1.0 & $14.0,15.1$ & 1.5 & 7.5 & 25.0 & 0.3 & -22.7 & 1 & 0.14 & 0.3 & 22.5 \\
\hline $14: 56: 08.60$ & $38: 00: 38.6$ & 1 & 0.283 & $16.5,14.1,16.2,16.0$ & 0.9 & $16.8,17.3$ & 2 & 7.5 & 24.9 & 0.1 & -23.8 & 1 & 0.61 & 0.1 & - \\
\hline $15: 01: 13.19$ & $23: 29: 08.2$ & 1 & 0.258 & $15.9,13.5,19.3,17.2$ & 1.9 & $17.4,17.6$ & 1 & 9.0 & 27.4 & 0.4 & -22.9 & 1 & 0.20 & 0.2 & 23.4 \\
\hline $15: 04: 20.74$ & $11: 52: 55.4$ & 3 & 0.171 & $16.7,15.0,19.5,17.4$ & 2.1 & $17.8,17.8$ & 1 & 5.5 & 26.7 & 0.6 & -21.6 & 1 & 0.10 & 0.2 & 21.6 \\
\hline $15: 07: 06.36$ & $-12: 25: 15.9$ & 1 & 0.185 & $15.3,12.6,18.2,17.1$ & 1.6 & $17.1,17.7$ & 1.5 & 8.0 & 27.5 & 0.3 & -22.5 & 2 & 0.64 & 0.3 & - \\
\hline $15: 10: 43.47$ & $-17: 35: 39.2$ & 1 & 0.151 & $15.9,13.9,17.9,16.9$ & 2.0 & $17.5,17.7$ & 1 & 7.5 & 27.4 & 0.4 & -21.6 & 1 & 0.20 & 0.3 & - \\
\hline $15: 13: 25.89$ & $46: 54: 07.9$ & 1 & 0.199 & $14.9,12.8,16.6,16.2$ & 1.1 & $16.5,17.5$ & 1.5 & 9.0 & 25.9 & 0.2 & -23.2 & 0 & 1.40 & 0.5 & 22.5 \\
\hline $15: 16: 53.24$ & $19: 00: 48.4$ & 1 & 0.190 & $13.5,11.4,15.8,14.5$ & 1.0 & $14.9,17.1$ & 3 & 8.0 & 26.7 & 0.2 & -24.8 & 3 & 6.60 & 0.2 & 21.7 \\
\hline $15: 17: 30.26$ & $-6: 33: 06.2$ & 1 & 0.128 & $15.8,13.7,17.0,16.3$ & 1.1 & $17.0,17.4$ & 1 & 7.0 & 26.3 & 0.1 & -21.7 & 1 & 0.45 & 0.5 & 22.2 \\
\hline $15: 17: 53.38$ & $17: 58: 44.1$ & 2 & 0.163 & $16.5,14.6,18.6,17.3$ & 1.7 & $17.2,17.3$ & 3 & 7.5 & 27.3 & 0.2 & -22.1 & 2 & 0.10 & 0.5 & 21.6 \\
\hline $15: 19: 01.50$ & $18: 38: 04.9$ & 1 & 0.187 & $16.4,14.3,18.9,17.5$ & 1.1 & $17.5,17.6$ & 1.8 & 8.0 & 26.2 & 0.3 & -22.2 & 2 & 0.10 & 0.2 & 21.7 \\
\hline $15: 21: 38.06$ & $-11: 59: 43.8$ & 1 & 0.153 & $15.6,13.6,18.2,17.3$ & 0.9 & $17.4,18.0$ & 1 & 8.5 & 27.0 & 0.3 & -21.8 & 0 & 0.74 & 0.5 & - \\
\hline $15: 21: 51.07$ & $22: 51: 20.8$ & 1 & 0.287 & $16.6,14.3,20.5,18.0$ & 1.6 & $18.3,18.4$ & 3 & 4.5 & 26.6 & 0.2 & -22.3 & 3 & 0.10 & 0.1 & 23.5 \\
\hline $15: 33: 49.87$ & $-3: 30: 34.7$ & 1 & 0.142 & $15.9,13.9,17.7,17.6$ & 1.2 & $18.0,18.3$ & 1.5 & 7.0 & 26.9 & 0.2 & -20.9 & 0 & 0.21 & 0.2 & 21.5 \\
\hline $15: 36: 03.17$ & $8: 01: 41.5$ & 3 & 0.120 & $16.1,13.9,16.6,16.0$ & 1.2 & $17.3,17.4$ & 1.2 & 8.0 & 27.3 & 0.3 & -21.3 & 3 & 0.10 & 0.4 & 21.3 \\
\hline $15: 45: 16.89$ & $38: 15: 39.3$ & 3 & 0.292 & $16.6,14.1,19.4,18.5$ & 1.2 & $18.3,18.6$ & 1.5 & 6.9 & 26.6 & 0.1 & -22.3 & 1 & 0.3 & 0.30 & 23.1 \\
\hline $15: 46: 53.53$ & $54: 57: 06.7$ & 2 & 0.107 & $15.5,13.4,17.1,15.8$ & 1.3 & $16.5,16.7$ & 3 & 7.1 & 25.6 & 0.2 & -21.8 & 0 & 0.26 & 0.4 & 23.5 \\
\hline $16: 37: 36.52$ & $25: 43: 02.8$ & 2 & 0.277 & $16.5,14.2,19.4,18.3$ & 1.5 & $18.3,18.9$ & 2 & 6.3 & 25.8 & 0.3 & -22.2 & 2 & 0.64 & 0.1 & 23.3 \\
\hline $21: 24: 41.63$ & $-17: 44: 45.8$ & 1 & 0.112 & $13.8,11.4,14.4,13.8$ & 1.2 & $15.3,16.0$ & 1 & 8.0 & 26.4 & 0.1 & -23.1 & 1 & 0.90 & 0.4 & 23.3 \\
\hline
\end{tabular}




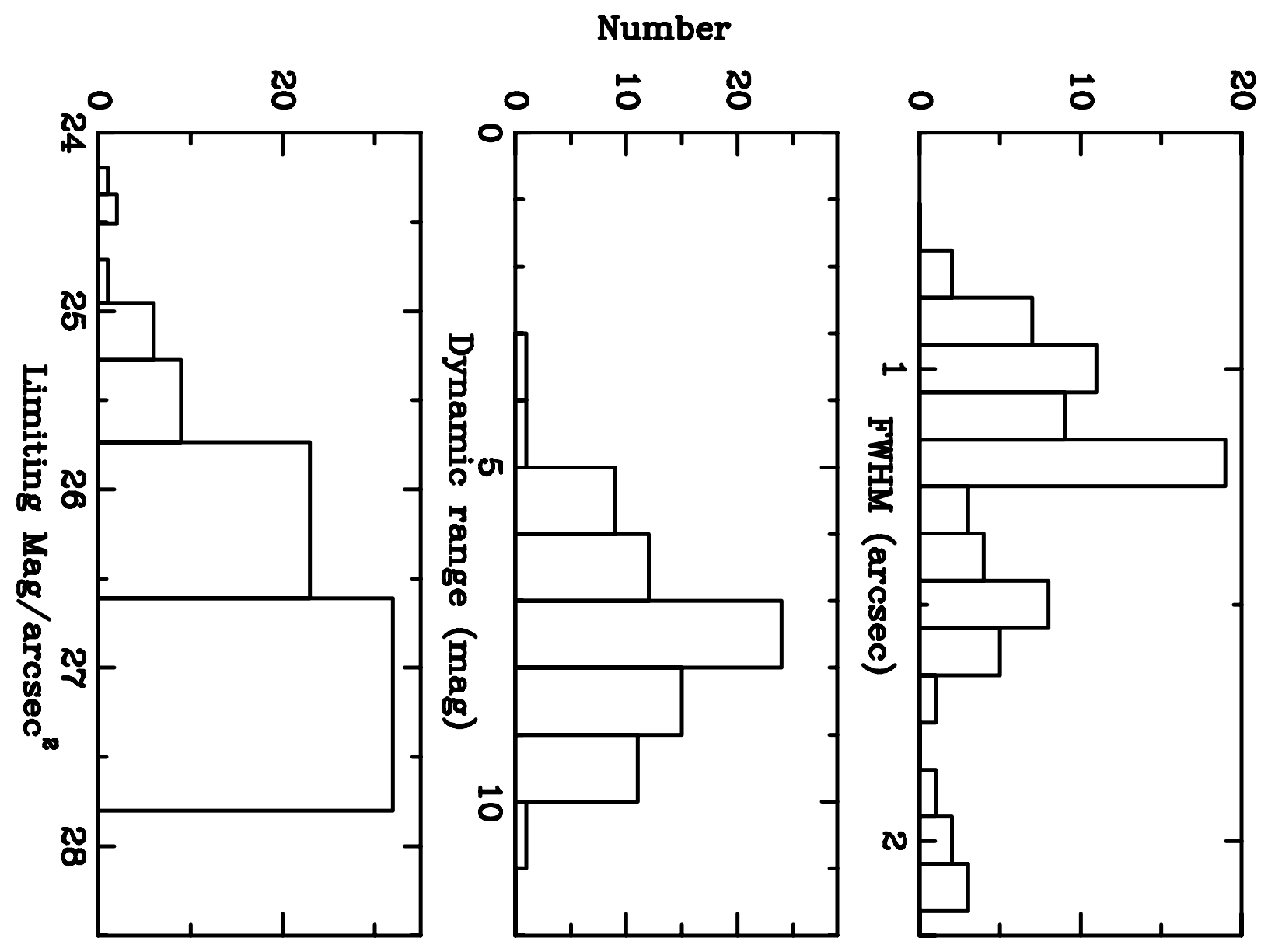


$\mathrm{K}$ magnitude

R magnitude

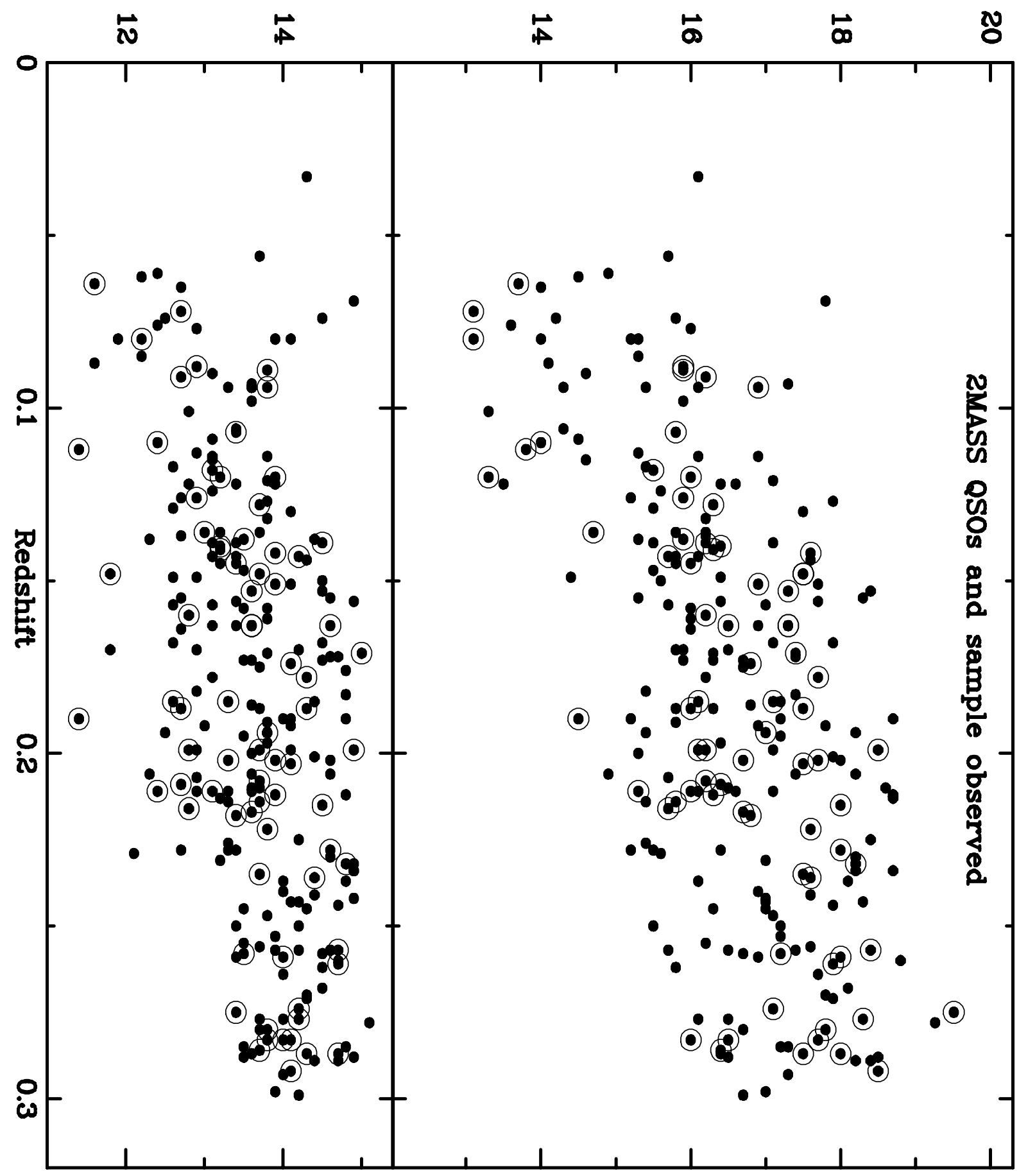




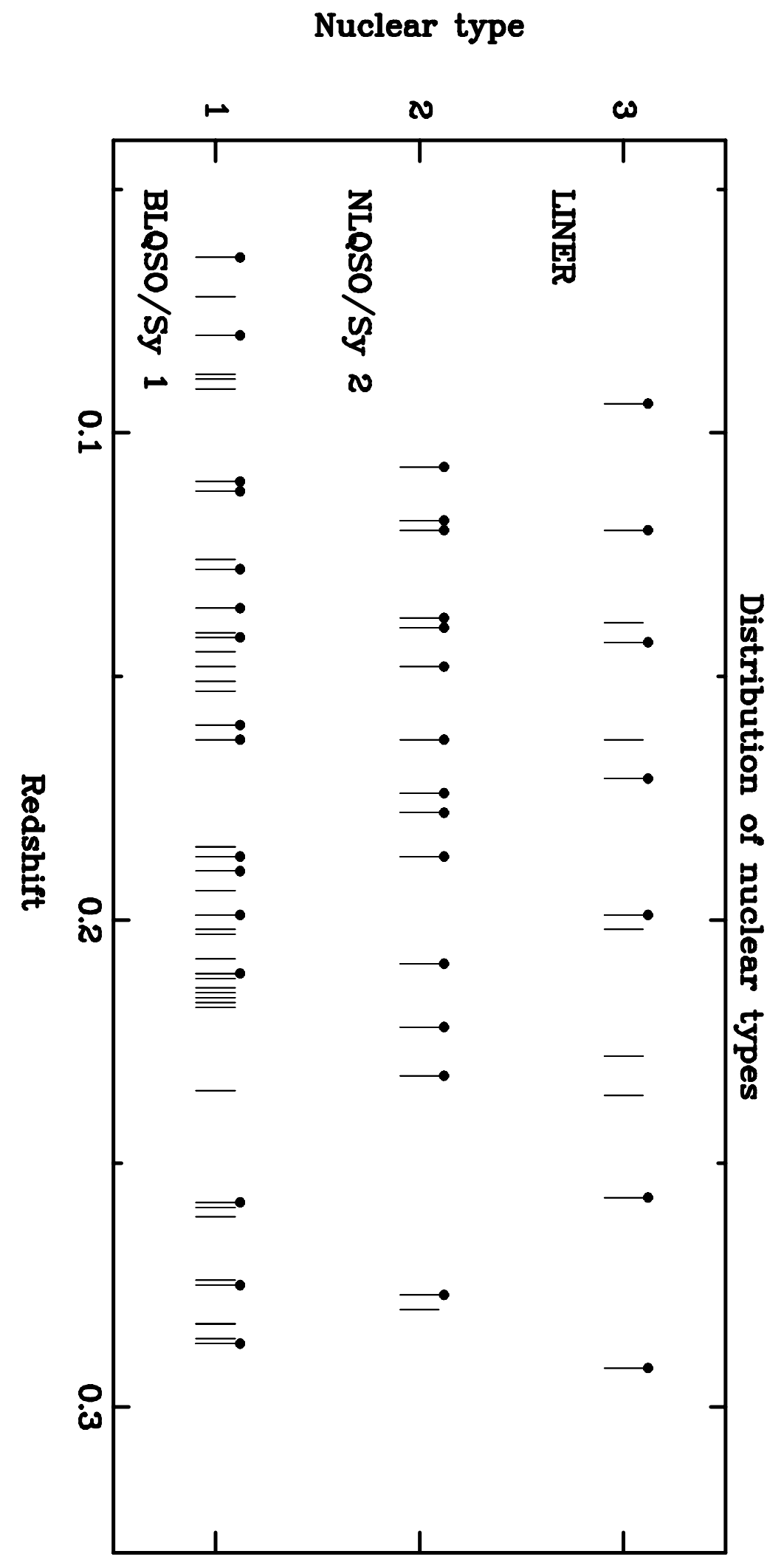



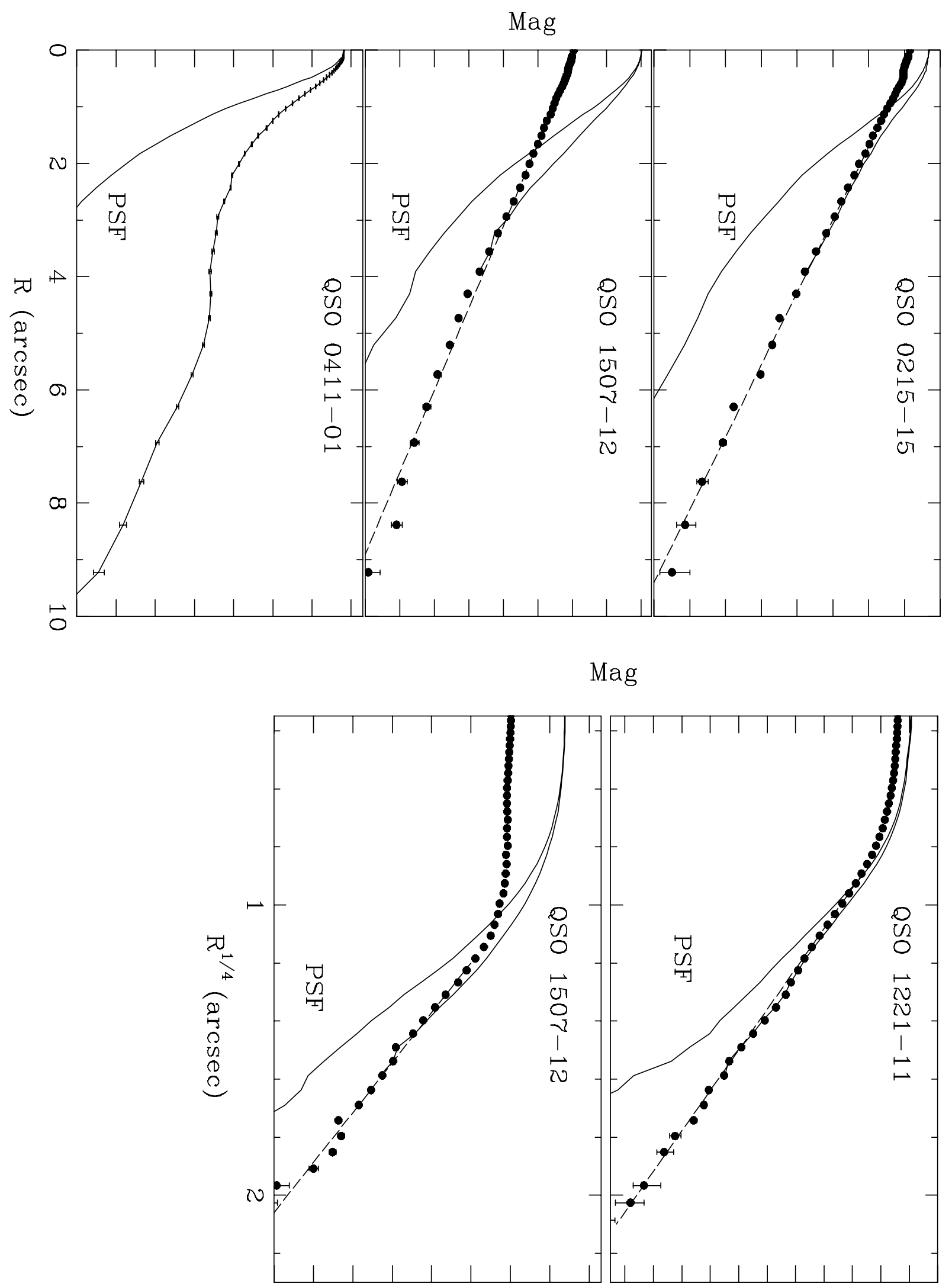


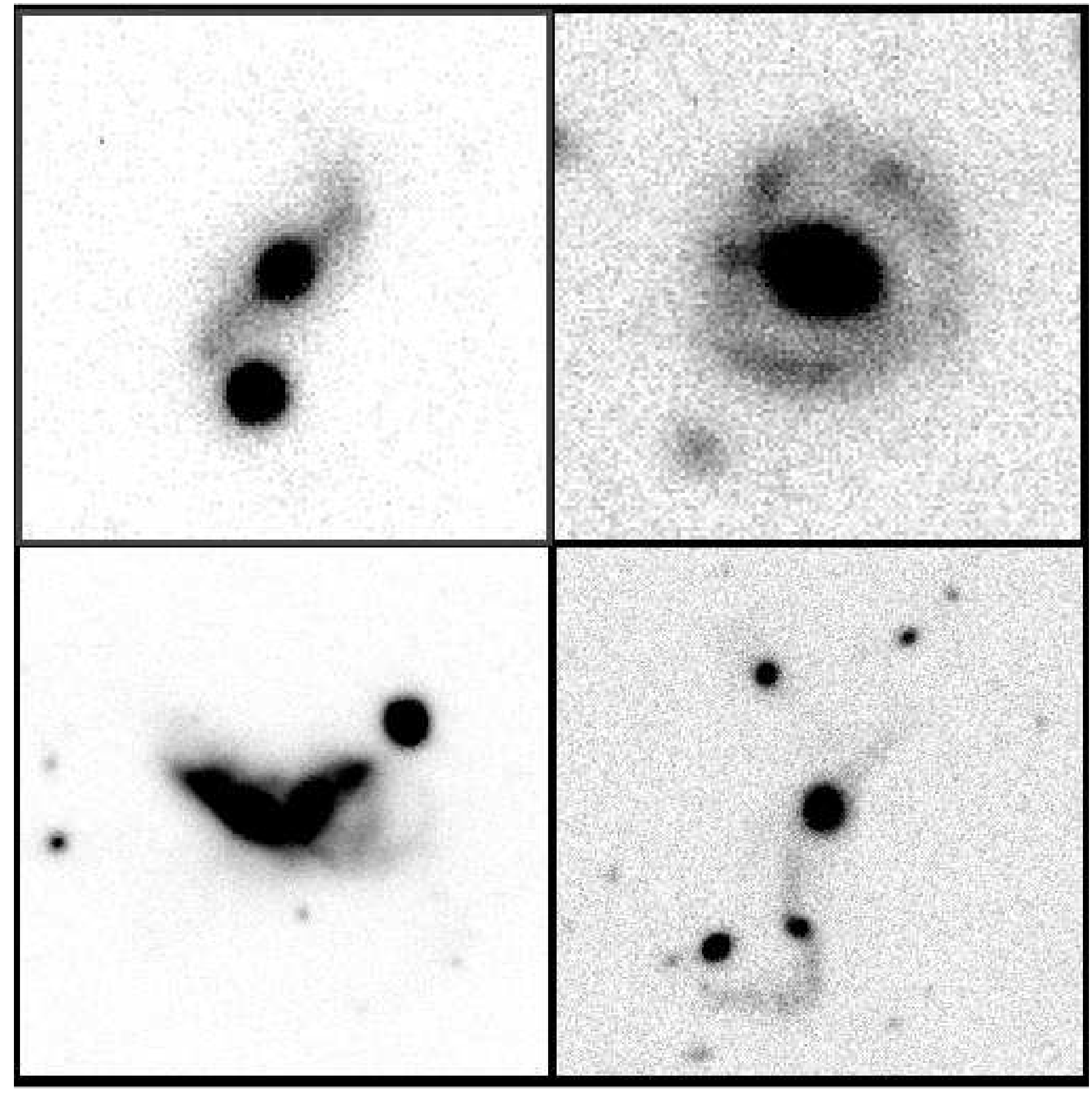




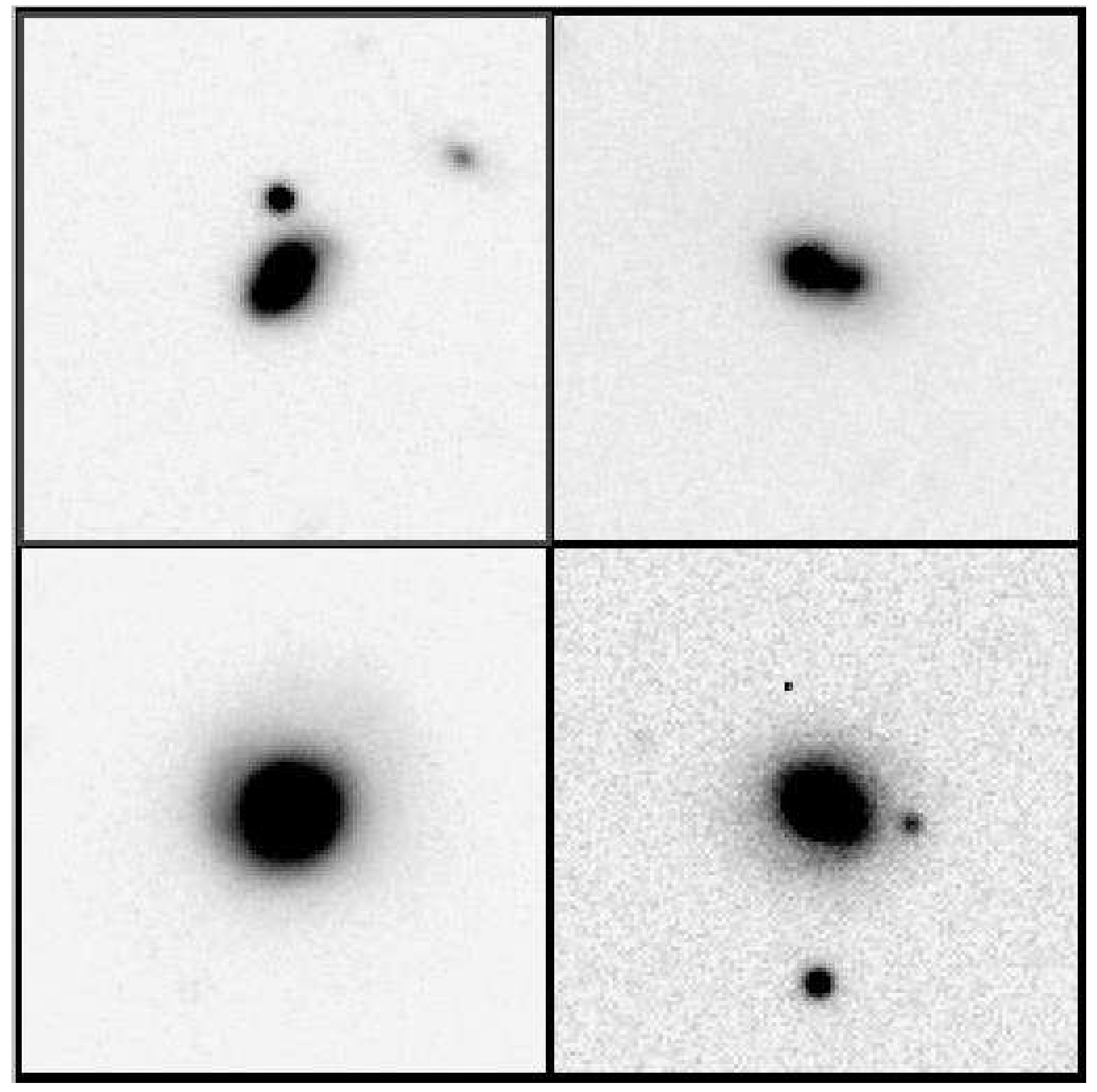




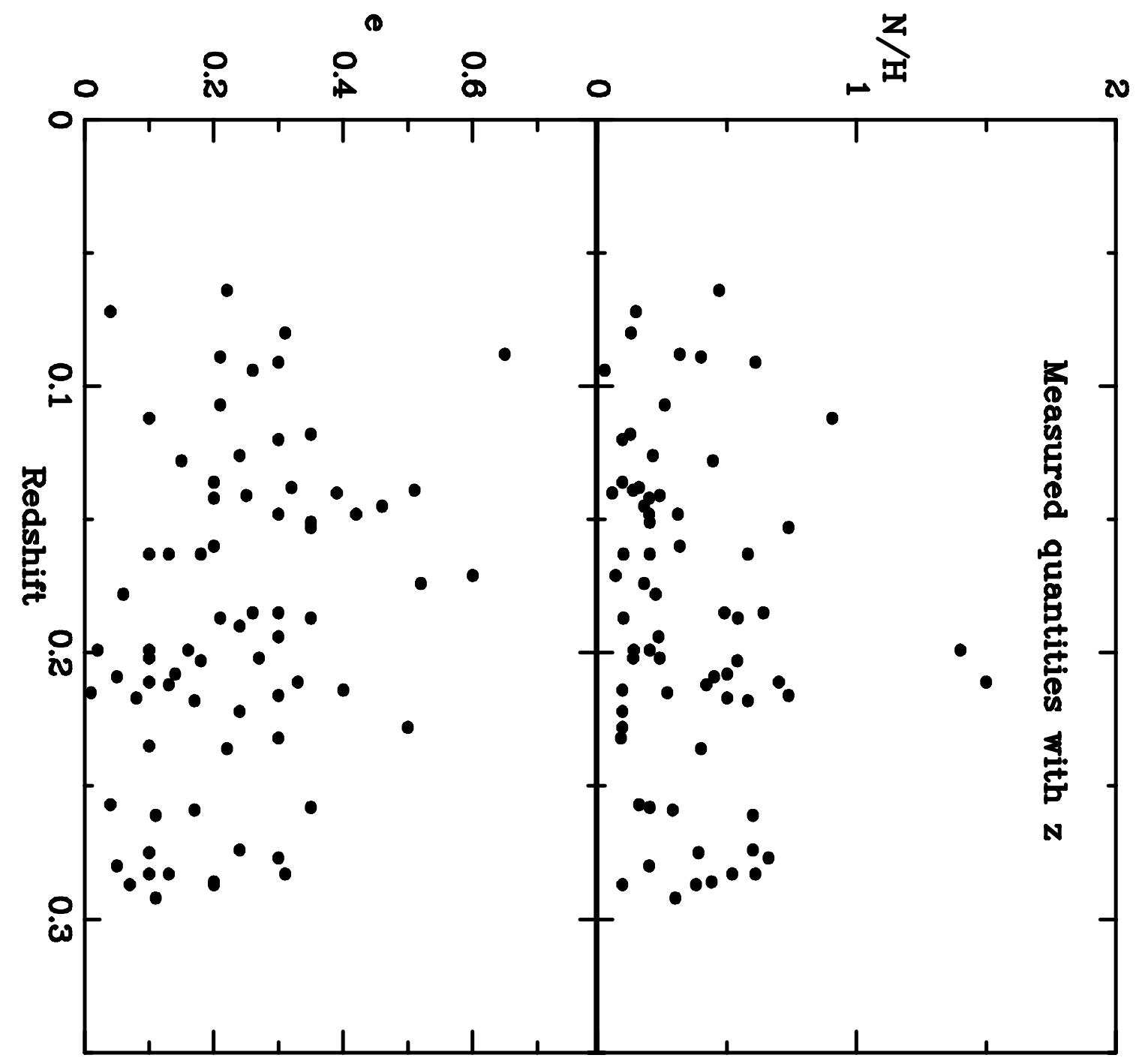




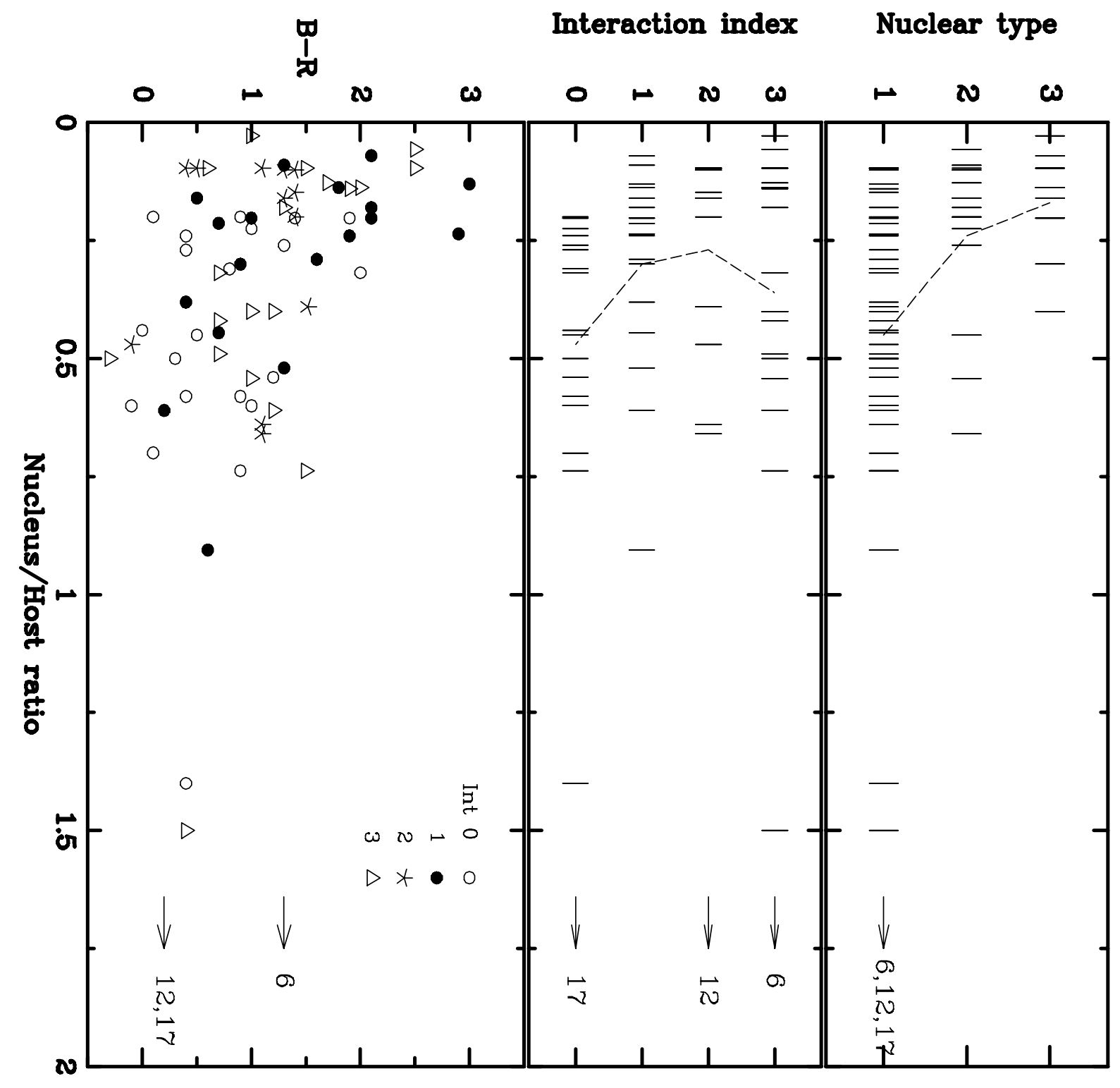




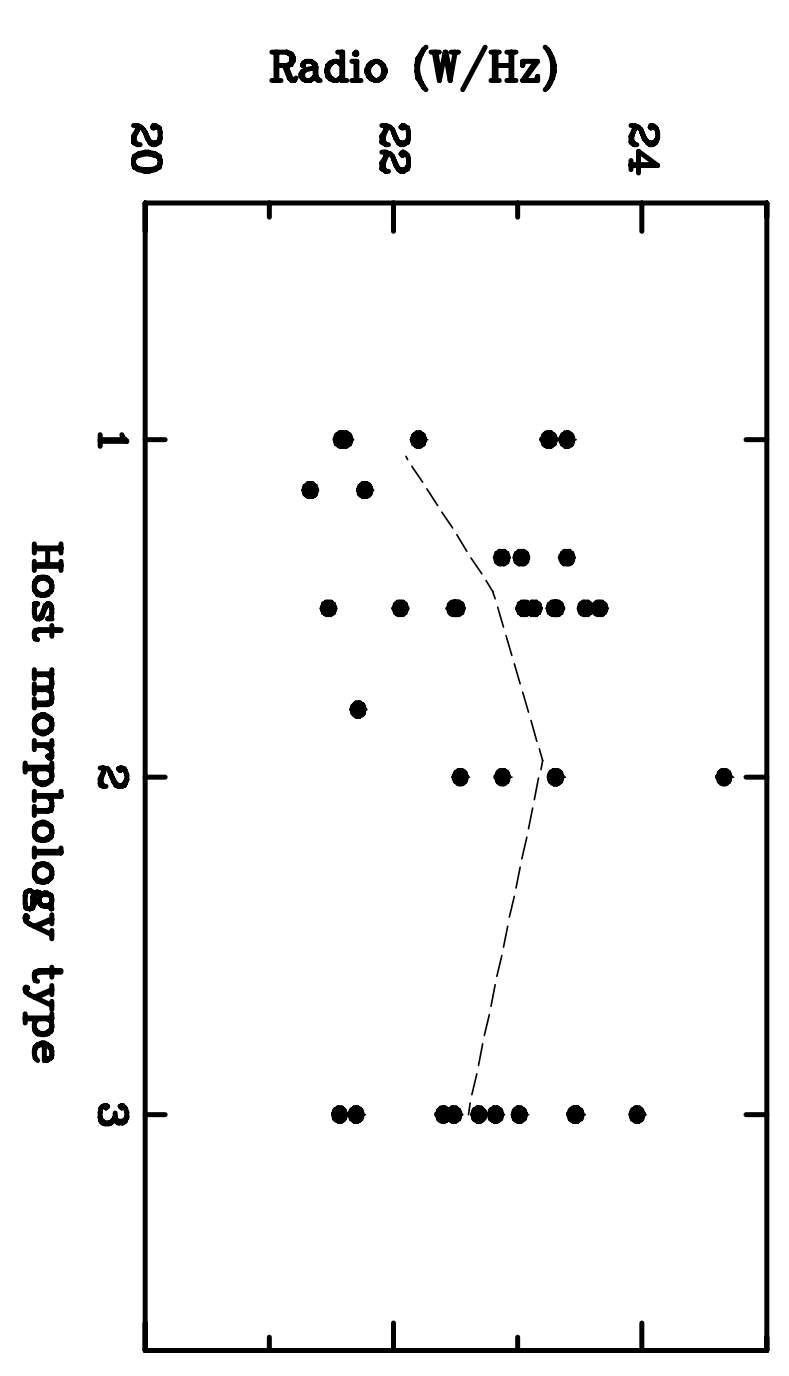

Host abs R mag

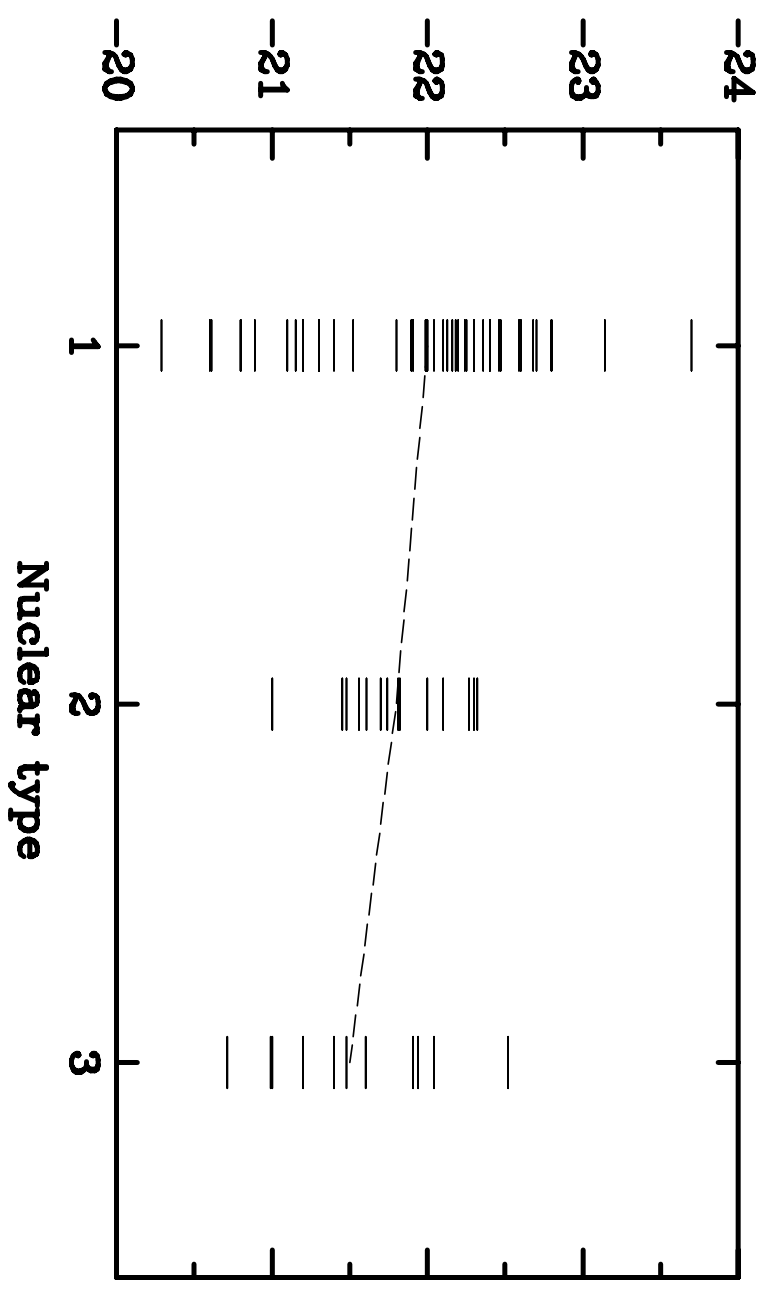

